\title{
Fuzzy multi-objective optimization design of fuel injector pump plunger spring
}

\author{
Erzhong An ${ }^{1, a}$, Yunchao Wang ${ }^{2, b}$
}

${ }^{1}$ College of Mechanical and Automobile Engineering, Jiangsu Teachers University of Technology, Changzhou, Jiangsu, 213001, China

${ }^{2}$ College of Mechanical Engineering, Jimei University,Xianmen,Fujian,361021, China

aanerzhong@yahoo.com.cn, bychaowang@jmu.edu.cn

Keywords: Fuel injector pump, Plunger spring, Fuzzy, Multi- objective optimization, Design

\begin{abstract}
Fuzzy theory and multi-objective optimization technology were applied to design fuel injector pump plunger spring based on consideration on its force condition. The mathematical model for the multi-objective optimization design was built under the objective of the maximal fatigue safety coefficient, the minimal weight and height, with the design variable of wire diameter $d$, mean diameter $D$ and working circle $n$. Then, the fuzzy solution of multi-objective optimization were use to solve the model. Practical example of calculation shows that, the plunger spring's weight, wire diameter and spring height increases a little, but the fatigue safety coefficient improves by $10.35 \%$ and $12.45 \%$ respectively, and the mean diameter reduced obviously.
\end{abstract}

\section{Introduction}

The plunger spring mainly bears alternating load during its working and its failure form is fatigue breaking. In practical operation, there is middle transition process from perfect available to full failure for spring with loads. Therefore, to design by using fuzzy method is more accords with the engineering practice. In the light of the design variable's randomness and fuzziness, a mathematical model for the multi-objective optimization design was established under the objective of the maximal fatigue safety coefficient, the minimal weight and height. Then, the fuzzy solution of multi-objective optimization were use to solve the model.

\section{Establishment of mathematical model of optimal design}

\section{Determine optimized variables}

The wire diameter $d$, mean diameter $D$ and working circle $n$ were chosen as design variables. The vector of decision variables is

$$
X=(d, D, n)^{T}=\left(x_{1}, x_{2}, x_{3}\right)^{T}
$$

\section{Establish objective functions}

Volume, mass and fatigue safety coefficient are all important performance indexes for spring, the maximum fatigue safety coefficient, minimum weight and height were selected as objective functions.

1)Maximize the fatigue safety coefficient [1]

In order to assure the spring strength and life, the maximum fatigue safety coefficient $S$ is the first optimized object.

$$
\max S=\left(\tau_{0}+0.75 \tau_{\min }\right) / \tau_{\max }
$$

Minimum and Maximum shear stress is

$$
\tau_{\text {max }}=8 K D F_{2} /\left(\pi d^{3}\right) \tau_{\text {min }}=8 K D F_{1} /\left(\pi d^{3}\right)
$$

Where $\tau_{0}$ is endurance limit at pulsating stress, $F_{1} 、 F_{2}$ is Maximum and minimum alternating load, $\mathrm{C}$ is spring index, $K$ is Wahl factor.

$K=(4 C-1) /(4 C-4)+0.615 / C \approx 1.66 /(D / d)^{0.16}, C=D / d$

For the convenience of calculation, the objective function can be converted to minimize its value 
(take the reciprocal value of function as optimized object).

$\min f_{1}(x)=\tau_{\max } /\left(\tau_{0}+0.75 \tau_{\min }\right)$

2) Minimize the spring weight

In order to achieve the minimum weight, the spring weight is the second optimized object. The weight of spring is given by the relation $w=\pi^{2} d^{2} D n \rho / 4$

Where $\rho$ is mass density of the spring, $\rho=7.8 \times 10^{-5} \mathrm{~N} / \mathrm{mm}^{3}$, therefore

$\min f_{2}(x)=1.925 \times 10^{-4} x_{1}^{2} x_{2}\left(x_{3}+2\right)$

3) Minimize the spring height

The minimum spring height is the third objective function.

$\min f_{3}(x)=H=\left(n+n_{2}\right) d=\left(x_{3}+2\right) x_{1}$

Where $n_{2}$ is inactive number of coils, $n_{2}=2$

\section{Constraint conditions}

The plunger spring should meet the strength and stiffness. Consider that there is middle transition process from perfect available to full failure for spring with loads, taking allowable stress, slenderness, spring index and the thresholds of various design variables as fuzzy subsets, the relevant design constrains are as follows:

1) strength condition

For ensuring the spring works normally, the largest shear stress $\tau$ in the wire must be less than or equal to the allowable shearing stress $[\tau], \quad \tau=8 K D F_{2} / \pi d^{3} \leq[\tau]$, thus

$g_{1}(X)=8 K F_{2} x_{2} / \pi x_{1}-[\tau] \leq 0$

2) stiffness constraint

$g_{2}(X)=F_{2}-\lambda G x_{1}^{4} /\left(8 x_{3} x_{2}{ }^{3}\right) \leq 0$

where $\lambda$ represents the maximum spring deflection, $G$ is shear elastic modulus.

3) spring index constraint

Usually spring index $\mathrm{C}$ is 4 to 18 , thus

$g_{3}(X)=4-x_{2} / x_{1} \leq 0$

$g_{4}(X)=x_{2} / x_{1}-18 \leq 0$

4) stability restraint

$g_{5}(X)=\left(\left(x_{3}+n_{2}-0.5\right) x_{1}+1.1 \lambda\right) / x_{2}-5.3 \leq 0$

5) non resonance constraint

In order to avoid resonance, the free vibration frequent $f\left(=3.56 \times 10^{5} d / n D^{2}\right)$ should be far away from the load frequency $f_{r}$, take $f \geq 10 f_{r}$.

$g_{6}(X)=f_{r}-0.1 f=f_{r}-0.356 \times 10^{5} x_{1} /\left(x_{3} x_{2}^{2}\right) \leq 0$

6) minimum working circle constraint

The number of active coils should be equal to or larger than minimum working circle $n \geq n_{\min }$

$g_{7}(X)=n_{\min }-x_{3} \leq 0$

7) mean diameter constraint

$D_{\min } \leq D \leq D_{\max }$

$g_{8}(X)=D_{\min }-x_{2} \leq 0$

$g_{9}(X)=x_{2}-D_{\max } \leq 0$

8) wire diameter constraint

$d_{\text {min }} \leq d \leq d_{\text {max }}$

$g_{10}(X)=d_{\min }-x_{1} \leq 0$

$g_{11}(X)=x_{1}-d_{\max } \leq 0$

9) Multi objective optimization design mathematical model

$x=(d, D, n)^{T}=\left(x_{1}, x_{2}, x_{3}\right)^{T}$ 


$$
V-\left\{\begin{array}{l}
\min f_{1}(x) \\
\min f_{2}(x) \\
\min f_{3}(x) \\
\text { s.t. } g_{i}(x) \leq 0 \quad j=1,2,3, \cdots, 11
\end{array}\right.
$$

\section{Determine subjection function}

There are several forms of membership functions. Echelon school function is often adopted as subjection function in engineering research[2]. To make the solution easier, the following linear subordinate function has been adopted.

1) For strength constraints

$$
\mu(x)= \begin{cases}1 & \left(x^{-j} \leq x\right) \\ \frac{x-x^{-j}}{x^{-1}-x^{-j}} & \left(x^{-1} \leq x \leq x^{-j}\right) \\ 0 & \text { (in other cases) }\end{cases}
$$

2) For the other constraints

$$
\mu(x)= \begin{cases}1 & \left(x^{-j} \leq x\right) \\ \frac{x-x^{-j}}{x^{-1}-x^{-j}} & \left(x^{-1} \leq x \leq x^{-j}\right) \\ \frac{x^{1} *-x}{x^{1} *-x^{j} *} & \left(x^{1} * \leq x \leq x_{j} *\right) \\ 0 & \text { (in other cases) }\end{cases}
$$

Where $\mathrm{x}^{-\mathrm{j}} 、 \mathrm{x}^{-1} 、 \mathrm{x}^{\mathrm{j}} 、 \mathrm{x}^{1}$ denote respectively the upper and lower bounds of interval, their value can be determined by using extend coefficient based on the common design standards and requirements. Limited by space, the computational process is omitted.

\section{Calculating optimization models}

The basic idea of fuzzy solving method for multi-objective optimization is as follows: The constrained optimum solutions for single objective are figured out and fuzzylized consequently. The set intersection of those solutions is then obtained. The required optimum solution for multi-objective optimization problem is the solution corresponding to the maximal membership function for the set intersection[3].

1) Find the constrained optimum solutions of single objective

$$
\left\{\begin{array}{l}
x=\left[x_{1}, x_{2}, x_{3}\right]^{T} \\
\min f_{i}(x) \quad(i=1,2,3) \\
\text { s.t. } g_{k}(x) \leq 0 \quad(k=1,2,3, \cdots, 11)
\end{array}\right.
$$

The maximum $M_{i}$ and minimum $m_{i}$ of single objective can be obtained by way of solving the model.

2) Fuzzy up the single object function

$$
\mu_{f_{i}}(x)=\left[\frac{M_{i}-f_{i}(x)}{M_{i}-m_{i}}\right]^{q}
$$

Where $q$ is non-negative real number, take $q=1 / 2$

3) Compute the membership function of fuzzy maximizing set

fuzzy maximizing set

$$
D=\bigcap_{i=1}^{3} f_{i}
$$

its membership function is

$$
\mu_{D}(x)=\wedge_{i=1}^{3} \mu_{f_{i}}(x)
$$


4) To search optimal solutions

$\mu_{D}\left(x^{*}\right)=\max \mu_{D}(x)=\max \hat{\mathrm{i}=1}_{\hat{f}_{i}}^{3} \mu_{f_{i}}(x)$

From (26) $x^{*}$ was derived, which is the optimal solution of multi -objectives fuzzy optimization. Therefore, multi-objective optimization model is converted to a single objective one.

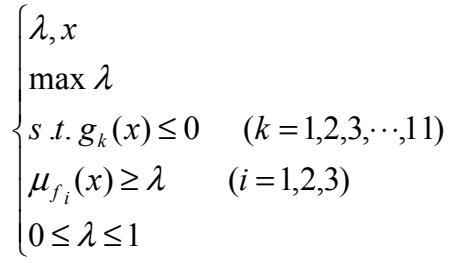

\section{Computation example}

A injection pump plunger spring is to be assembled with a preload of $260 \mathrm{~N}$ and will operate with a maximum load of $585 \mathrm{~N}$ at $20 \mathrm{~Hz}$. Spring material is $50 \mathrm{CrVA}$, oil quenching and tempering. The lift of the cam is $9.5 \mathrm{~mm}$, the preferred range of spring mean diameter is $18 \leq D \leq 34$, number of active turns or coils is $6 \leq n \leq 12$, wire diameter is $2.5 \leq d \leq 7.5$,spring index $C \geq 6$. Find out the spring with maximum fatigue safety coefficient, minimum weight and height.

Table 1 shows the comparison of the results for the injection pump plunger spring design optimization problem. It can be seen from Table 1, that compared with the nominal design and regular optimization the wire diameter and spring height increase by $3.64 \%$, spring weight also increases a little, but the fatigue safety coefficient improves by $10.35 \%$ and $12.45 \%$ respectively, and the mean diameter reduced obviously. The effect after optimize is obvious.

Tab.1 Result comparison of fuzzy optimization with regular optimization and nominal design scheme

\begin{tabular}{|lcccc|}
\hline \multicolumn{1}{|c}{ Parameter } & $\begin{array}{c}\text { nominal } \\
\text { design }\end{array}$ & $\begin{array}{c}\text { regular } \\
\text { optimization }\end{array}$ & $\begin{array}{c}\text { fuzzy } \\
\text { optimization }\end{array}$ \\
\hline Wire diameter & $d(\mathrm{~mm})$ & 5.5 & 5.5 & 5.7 \\
\hline mean diameter & $D(\mathrm{~mm})$ & 32 & 33 & 31 \\
\hline number of the working coils & $n$ & 6 & 6 & 6 \\
\hline fatigue safety coefficient & $S$ & 1.2017 & 1.225 & 1.3516 \\
\hline spring weight & $w(\mathrm{~N})$ & 1.5474 & 1.4907 & 1.5511 \\
\hline Spring height & $H(\mathrm{~mm})$ & 44 & 44 & 45.6 \\
\hline
\end{tabular}

\section{Conclusions}

1) The mathematical model of multi-objective optimization design for fuel injector pump plunger spring was established based on consideration on the fuzziness of design variables and constrains, which can best meet the engineering practices. Then, the fuzzy solution of multi-objective optimization were used to solve the model.

2) The optimal results show that by using multi-objective optimization method the fatigue safety coefficient improves by $10.35 \%$ and $12.45 \%$ respectively, compared with regular optimization and nominal design. Although the wire diameter and spring height increase a little, it has almost no effect on engineering practice.

3) This method can improve the design quality and efficiency, and it has some engineering practical value.

\section{Acknowledgements}

This work was financially supported by the Fujian Natural Science Foundation (2010J05118), the China National Science Foundation (51105171) and the Foundation for young professors of Jimei University, China(2011B001) 


\section{References}

[1] Weixin Liu. Mechanical optimal design[M].Beijing: Tsinghua University Press, 1994. In Chinese [2] Shangming Liang, Guofu Yin. Modern machine design technique [M].Beijing: Chemical Industry Press,2005.5. In Chinese

[3] Qingsheng Xie,Yanke Luo,Qi Li. Mechanical engineering fuzzy optimization method [M]. Beijing: China Machine Press, 2002,5. In Chinese

[4] Hongzhong Huang.The machine design fuzzy optimization principle and apply[M]. Beijing: Science Press, 1997. In Chinese

[5] Xiaojin Fu. Spring fuzzy reliability optimization design[J].Mechanical design \& Manufacture, 2000,37(1):4-6. In Chinese

[6] Bingliang Ye, Gaohong Yu,Yun Zhao. Multi-objective fuzzy reliability optimization design of tractor's final transmission[J].Transactions of the Chinese society for agricultural machinery, 2010,41(4):95-100. In Chinese 